\title{
Spring diagrams for the visualization of construction project dynamics
}

\author{
Vadim Undozerov* \\ Moscow State University of Civil Engineering, Yaroslavskoe shosse, 26, Moscow, 129337, Russia
}

\begin{abstract}
Construction projects are very dynamic, so change management is crucial there. Changes in schedule and in stakeholders relationships are among the most important change management areas. Making decisions on such changes is an extremely difficult task. It can be facilitated by special visualization techniques. The existing techniques are analyzed and their drawbacks revealed. In order to address these drawbacks, new techniques are proposed for the visualization of both schedule and stakeholders relationships changes. They are based on the application of the spring image used in mechanics to the visualization of the project's dynamics. The performance of the techniques is shown in examples. The results are discussed and further research is outlined.
\end{abstract}

\section{Introduction}

Construction projects are very dynamic in nature. During the execution stage, many changes are made. The success of a project depends highly on the quality of change management [1]. In this paper we will focus on the management of changes in activity scheduling (rescheduling) and in stakeholders relationships. These changes are very important because they contribute significantly to project stability.

Construction schedules are often highly volatile during execution [2], especially at an operational level [3]. There are different types of changes such as increasing or decreasing activities' expected duration, interrupting, postponing the start of an activity, as well as adding new ones, e.g., rework in order to eliminate defects [4]. The need to make changes is related to impossibility or inexpediency of following the original plan $[2,5]$. It might be due to changes in project requirements [6], poor execution requiring rework [4], delivery delays, etc. $[7,8]$.

Changes in one activity cause changes in others which are directly or indirectly related to it. In complex projects which are characterized by a large number of interrelated tasks, multiple stakeholders and high uncertainty, changes even in one activity can lead to significant ramifications for the project as a whole [4, 9]. These ramifications can be either positive (for example, reducing the duration of a critical path activity decreases the duration of the whole project), or negative, even up to failure [3,4]. Failure is referred here as the refusal to continue the project or significant time and/or cost overruns if the project has been still completed [4].

An example of changes' negative ramifications is work quality deterioration due to socalled "schedule pressure" [4], i.e., deadline overreduction, which is a decision often used to compensate for delays. Poor quality necessitates rework, the time lost on it is compensated again by pressure on performers, which leads to new quality problems and,

\footnotetext{
* Corresponding author: und-vadim@yandex.ru
} 
consequently, to the need for new corrections [4]. Thus, a "vicious cycle" occurs that can destabilize the project and lead it to collapse [4]. Of course, such a development can be prevented by increasing workforce, but such an increase faces a number of constraints, such as lack of space $[10,11]$. Other negative consequences of changes are related to violation of stakeholders' own plans, which leads to inefficient resource allocation, conflicts [2], and other problems.

Stakeholder relationships are also very dynamic. The list of stakeholders and their attitudes toward other project participants and to the project as a whole change during project realization. Tension and conflicts between stakeholders occur due to unmet expectations, lack of resources, uncertainties, etc. Typically, after one problem has been solved, there is a period of stability. Then, however, new problems occur, bringing a new round of contention between stakeholders before resolution. In complex projects with dozens and even hundreds of stakeholders (subcontractors, vendors, etc.) there are many such cycles going on simultaneously with different rhythms. It makes the project environment difficult to manage.

Managers should constantly account for and predict changes in stakeholders relationships, because they significantly impact a project's stability and effectiveness [12]. The low quality of stakeholders relationships management can lead to disastrous consequences. It is proved to be one of the most important contributors to projects' failure [13]. As in the case of scheduling, in the sphere of stakeholder's relationships "domino effect" also takes place. C. Eden, T. Williams, F. Ackermann and S. Howick [3] illustrated it with a simple but very expressive example in which too risky behavior of one of the participants in an appartement's interior facilities leads to big time and cost disruption. The authors showed how a small problem, uncertainty about required sockets' arrangement, involves first this participant, then, gradually - almost all others into imprudent and negligent performance.

Thus, project management effectiveness is largely determined by the quality of decisions on changes in schedules and on stakeholders relationships management. In complex projects, making such decisions is an extremely difficult task, since it requires accounting for multiple stakeholders' interests and expectations, rational allocation of various resources, and forecasting the immediate and long-term consequences of the decision [3, 4]. The situation is exacerbated by the non-linearity of factors affecting a project. It means that the events that are insignificant individually may lead to large and often hardly predictable consequences in the context of the whole project [4, 9]. Furthermore, it is difficult to formalize some factors, such as staff qualification and motivation, so in practice they are taken into account by managers intuitively to a certain extent.

\section{Methods}

One of the most effective approaches to facilitate decision-making is visualization. Visualization allows to present large amounts of information in a quite simple, intuitive form. There are many visualization methods used in project management $[14,15]$. In scheduling, the Gantt chart and network diagrams (used in CPM, PERT, GERT methods) are the most common $[15,16]$. Stakeholders' interaction visualization includes such methods as various types of graphs [12], "The Stakeholder's Circle" [17, 18] and "The Organizational Zoo" [17, 19].

However, the existing visualization methods are not convenient for the dynamic, everchanging environment of real construction project execution stage. This drawback will be described further and the new two visualization techniques will be presented as an attempt to address this issue. The first technique is oriented to scheduling, the second one is for stakeholders relationships visualization. Both are based on the same idea that springs 
images used in mechanics can be also convenient for construction dynamics representation. This assumption stems from the natural ability of these images to create an illusion of tension and relaxation which is important when making decisions.

\section{"The spring chart" for scheduling visualization}

The scheduling visualization methods are mainly aimed at visualization at the project design stage, helping designers to create a process structure, allocate resources, identify critical path, etc., and are not very convenient for rescheduling. Based on the schedule created at the $n$-th change, it is impossible to find out anything about previous changes, as well as about the degree of activities durations' deviation from the initial schedule. That contributes to making decisions without taking into account previous changes. For example, if one needs to reduce the backlog, he/she may decide to increase schedule pressure, while it is already critical due to the early decisions. This can lead to project destabilization by "vicious cycle", as described earlier [4]. Thus, it is necessary to develop visualization methods with "accumulation", in which each new graph reflects the "trace" of previous changes and deviations from the original plan.

As such a method, the Gantt chart modification is proposed by the author, in which each activity-representing bar is replaced by the image of a spring used in mechanics (Fig. 1). This image is a simplified projection of a real spring on a plane parallel to its axis. This projection is a jagged polyline (the projection of a cylindrical helix - spring's geometric shape). The distance between the spring's start and end points ("axis length") is proportional to the activity's duration $(t)$, similar to the length of a bar in the Gantt chart. Therefore, it can be denoted just as $t$ and measured conditionally in units of time ("months", "days", etc.), implying the corresponding spatial units (e.g., "month" may mean $1 \mathrm{~cm}$ of "axis length"). The same applies to other spatial parameters in the chart - all of them will be conditionally expressed here in the corresponding time units. The distance between the nearest intersection points of a "spring" with its own axis ("step" $x_{0}$ ) is constant. It is convenient to make the step equal to a duration unit. The "teeth" of the spring (the turns of the helix in the projection) alternate staggered on the sides of the axis. The distance between the axis and the teeth' vertices $(h)$ is constant, the projection of a vertex on the axis divides a step in half. In the original graph, the angle at the top of the teeth is $90^{\circ}$ (Fig. 1a), which is meant to be perceived as equilibrium. At the first change in the graph, the springs are "compressed" and "stretched" - a step decreases/increases in proportion to the reduction/increase in the activity's duration (Fig. 1b). For the second change, $x_{0}$ and $t_{0}$ is assigned the values of the step and duration after the first change (respectively $x_{1}$ and $t_{1}$ ), the resulting step and duration is assigned the index 2 . Then the procedure is repeated, in general we have an iterative process:

$$
x_{i}=x_{i-1} \cdot \frac{t_{i}}{t_{i-1}}, \quad i=1,2, \ldots
$$

where $t_{i}, x_{i}$ - respectively the activity duration and the step after the $i$-th change; $t_{0}, x_{0}$ - respectively the activity duration and the step in the initial schedule.

The spring's shape (the number of teeth, "diameter") remains the same, only axis length changes, which creates an illusion of "compression" or "stretching". The decision-making manager can see which activities deviate from the original schedule and how much. This helps him/her to determine which activities should not be reduced, since they have already been significantly compressed, and which ones should be given a particular attention to due to huge delay. 
(a)

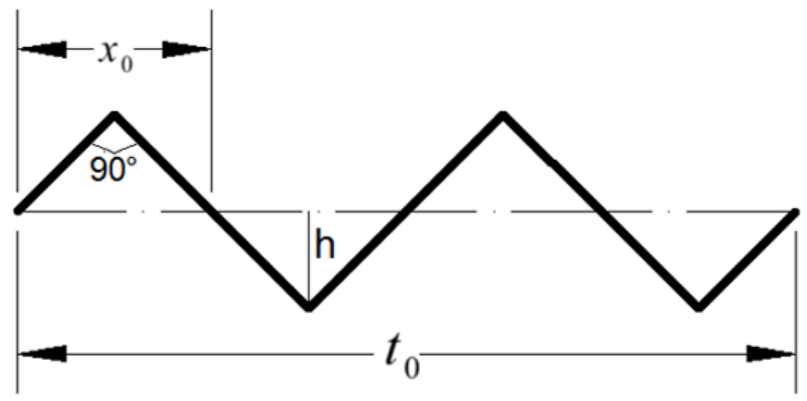

(b)

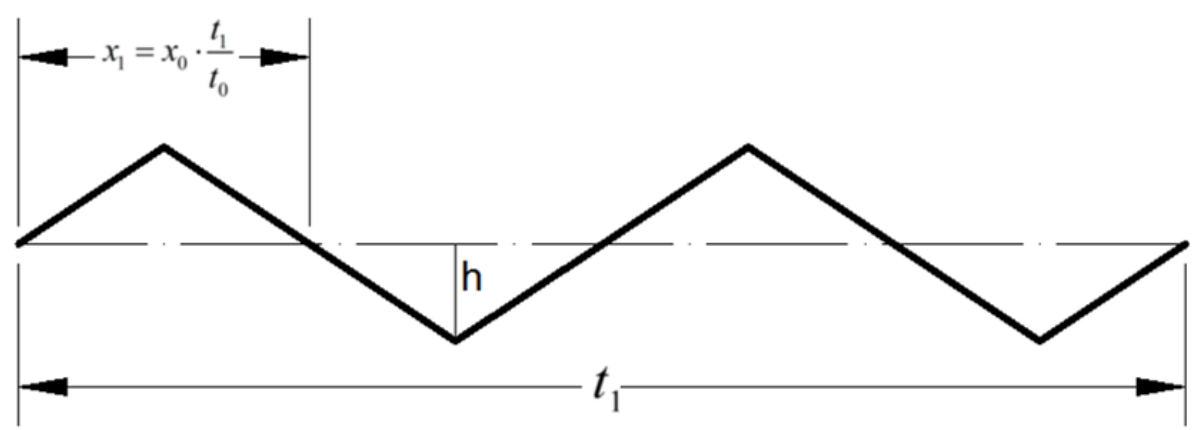

Fig. 1. "The spring chart" technique for schedule dynamics' visualization. Activity image in the form of spring (instead of the Gantt chart's bar): a) in the initial schedule; b) after the first change.

"The spring mapping" for stakeholders' interactions visualization

The existing techniques for stakeholders relationships visualization can be divided into two types.

The fist can be named "connections-oriented". It abstracts from the stakeholders' inner properties (such as level of discontent, expectations, fears, behavioral pattern, etc.) and represents just how stakeholders are connected with each other. "Connection-oriented" techniques are based on graphs in which the nodes represent stakeholders and the edges represent communications between them.

The second type can be named "stakeholder-oriented". Oppositely to "connection oriented" techniques this type focuses on stakeholders' inner properties and abstracts from connections. An example of these techniques is "The Stakeholder Circle" in which stakeholders are represented as the sectors of a circle. The stakeholder's properties (influence, urgency, engagement level, etc.) are shown by position of the sector, its color and pattern. Another example, "The Organizational Zoo", is an ingenious technique which represents stakeholders as animals (lions, mice, unicorns, etc.), to give a quick insight into their behavioral pattern. Though quite comical, at first glance, such approach proved to be useful in naming participants in stock market ("bulls", "bears", etc.) [20] and political sphere ("hawks and doves", "hedgehogs and foxes", etc.) [21].

The existing techniques have the common drawback that they are not convenient when stakeholders relationships change frequently. From the graphs as well as from "The Stakeholder Circle" it is difficult to get the history of stakeholders properties' previous states. And it is funny to imagine a lion turning into a sheep in "The Organizational Zoo". In order to create the visualization tool that would be able to give a deeper insight into the dynamics of stakeholders relationships, the same idea of the spring image implementation was used (Fig. 2). 
Every stakeholder is represented as a spring. The level of "a spring's tension" (i.e., the step's length) is proportional to the psychological tension of a person (Fig 2a). In simplified model there can be 3 states - equilibrium, anxiety and half-reassurance. Equilibrium takes place when work is going on as planned and there is nothing to stop it, as well as there are no conflicts between stakeholders. Anxiety emerges when some problem occurs, e.g., shortage of materials or a conflict between stakeholders. Half-reassurance usually takes place after the problem has been solved but temporarily or partly. Equilibrium can be represented as the spring with the $90^{\circ}$ angle at the top of the teeth, anxiety - as the spring of the same length (as the person is the same), but with the step three times shorter than equilibrium, and half-reassurance - the same length and two times shorter than equilibrium. The mapping is rendered by connecting the interacting stakeholders' springs at one point. In the Fig. $2 \mathrm{~b}$ the map of 5 interacting stakeholders is shown. The stakeholders 1 and 2 are anxious (maybe they are in conflict with each other), the stakeholders 3 and 4 are in equilibrium state and the stakeholder 5 is half-reassured.

a)
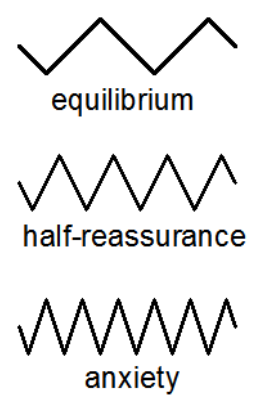

b)

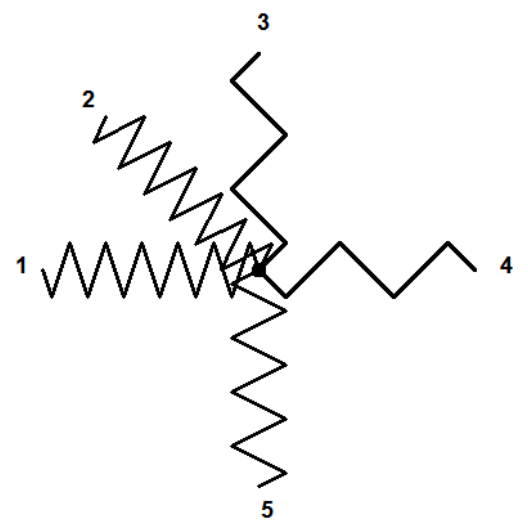

Fig. 2. "The spring mapping" technique for stakeholders relationships' dynamics visualization. Stakeholders are represented as springs which "tension" corresponds with their psychological tension (a). Springs representing interacting stakeholders join together at one point (b).

\section{Results}

The performance of the new visualization techniques is shown in two examples. The first example is an arbitrary schedule consisted of 5 activities, and "the spring chart" is used to visualize its dynamics during execution. The second example is a case described in [3] in order to illustrate the idea of "domino effect" in construction. "The spring mapping" is used to visualize the stakeholders relationships development in this case.

"The spring chart" implementation (an example)

As mentioned above, the schedule consists of 5 activities. According to the initial plan (Fig. 3 above), Activity 1 lasting 4 months opens the project, Activities 2 (5 months) and 3 (3 months) begin immediately after the end of Activity 1, Activity 4 (3 months) follows Activity 3, and the final Activity 5 (4 months) begins only when all the previous ones are completed. The step for all activities is assumed to be 1 month. During the Activity 1 execution, it was found that productivity had been overestimated and the expected completion of Activity 1 would not be 4, but about 6 months. That necessitates rescheduling in order to prevent the overall project deadline's disruption. The graph after the change is shown at the bottom of Fig. 3. To alleviate risks, it was decided to reduce the duration of the activities which follow Activity 1 directly: Activity 2 - from 5 to 4 months, Activities 3 and 4 - from 3 to 2 months. The corresponding steps after the change are 
(according to the equation (1)): Activity 1: $1 \times(6 / 4)=1 \times 1,5=1,5$ (months); Activity 2 : $1 \times(4 / 5)=1 \times 0,8=0,8$ (months); Activities $3,4: 1 \times(2 / 3)=0,66$ (months).

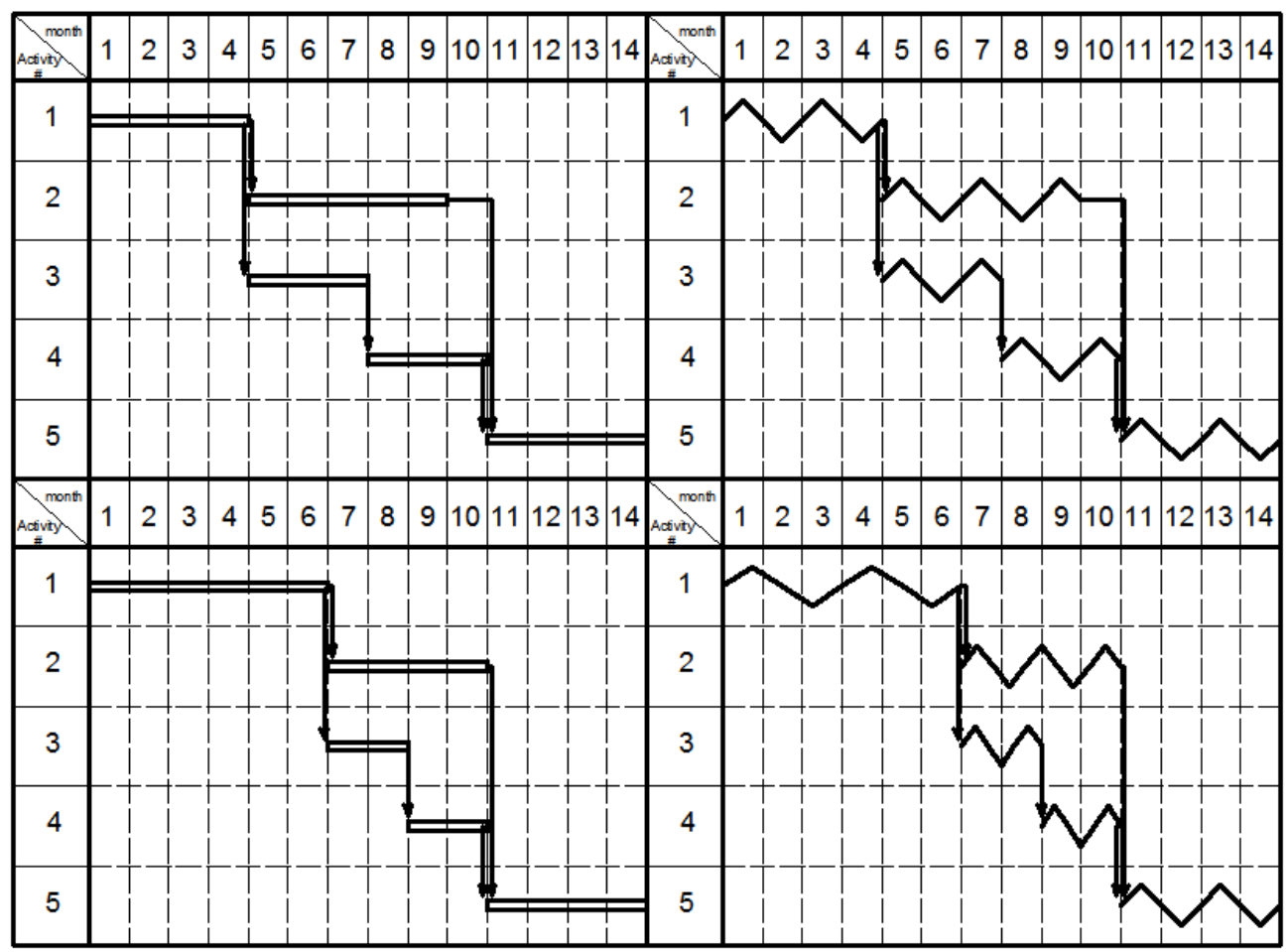

Fig. 3. "The spring chart" visualization technique implementation (an example). At the left - the traditional, at the right - the modified Gantt chart. At the top - the original plan, at the bottom - the plan after rescheduling.

\section{"The spring mapping" implementation (an example)}

C. Eden, T. Williams, F. Ackermann and S. Howick [3] used an interesting example to explain how small disruptions can lead to great ramifications. This example, illustrated with "the spring mapping" technique, will be presented further (Fig. 4).

There is a goal to make some modifications to a bedroom, kitchen and bathroom. At the onset one person works in each premise - a carpenter in the bedroom, a plumber in the kitchen and an electrician in the bathroom. There is enough space to everybody, all know what to do and have everything needed, so the activities are going on as planned and everybody is calm. This state of "equilibrium" is shown in Fig. 4a. Alongside with the 3 springs representing the already known participants, there is the fourth spring which represents a consumer, who also plays a role of manager in this case.

Suddenly the situation changes. The carpenter realizes that he can't finish his work in the bedroom (the floor), because some cables are supposed to be laid under it, but the manager hasn't provided any information needed to do it. The carpenter becomes uneasy (Fig. 4b). After the futile attempts to force the manager, the carpenter decides to take a risk and makes a part of the remaining floor accordingly to his own intuition. As it is only a trade-off, he remains somewhat anxious, though less than before (Fig. 4c). 
a)

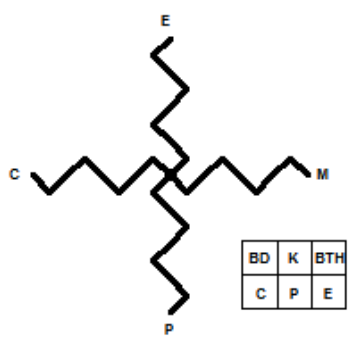

d)

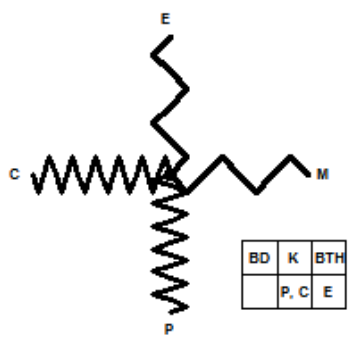

g)

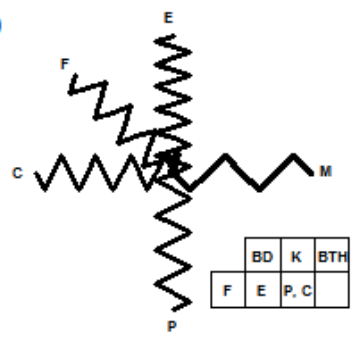

b)

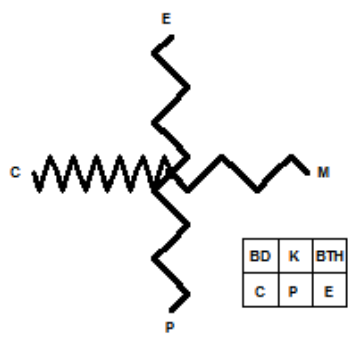

e)

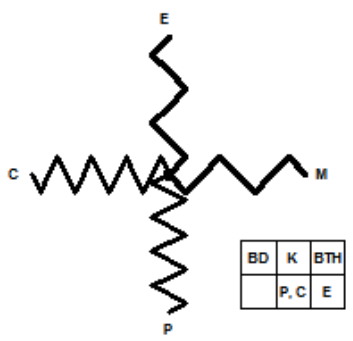

h)

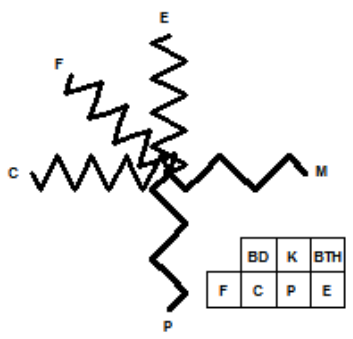

c)

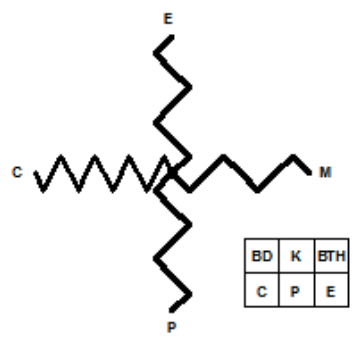

f)

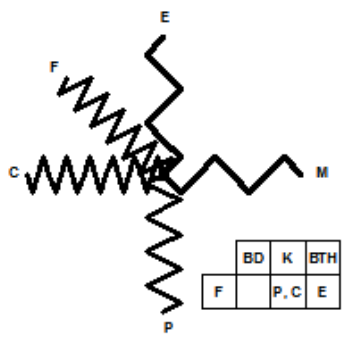

i)

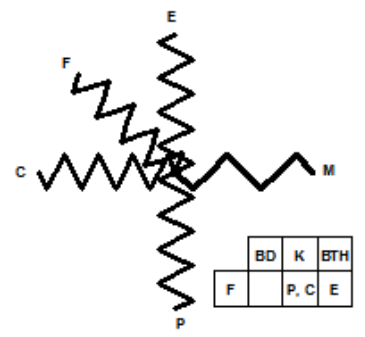

Fig. 4. "The spring mapping" visualization technique implementation (an example). a-i - the 9 successive participants' psychological states. $\mathrm{C}$ - the carpenter, $\mathrm{P}-$ the plumber, $\mathrm{E}-$ the electrician, $\mathrm{M}$ - the manager, $\mathrm{F}$ - the carpet fitter, $\mathrm{BD}$ - the bedroom, $\mathrm{K}$ - the kitchen, BTH - the bathroom.

Having nothing to do more in the bedroom, he goes to the kitchen to fit the furniture. But as he has left the bedroom earlier than planned, there is the plumber still working in the kitchen. A conflict between two occurs (Fig. 4d) since overcrowding impedes normal work. Finally, the plumber concedes but both are still quite discontent due to inconvenient conditions (Fig. 4e).

The situation exacerbates when a new worker, a carpet fitter, arrives expecting the bedroom floor completed. He gets angry when he sees that it is not the case, and the conflict between him and the carpenter occurs (Fig. 4f).

The carpenter has to persuade the electrician to leave his work in the bathroom and to lay the cabling in the bedroom intuitively. This trade-off causes a half-reassurance for the carpenter and the carpet fitter, but makes the electrician angry (Fig. 4g). This point is interesting, because now all the participants are uneasy to one extent or another.

After completing the cabling in the bedroom, the half-reassured (not fully, as there is doubt about the result's correctness) electrician comes back to the bathroom. The carpenter leaves the kitchen (in the plumber's relief) and finishes the bedroom floor. This situation is shown in Fig. 4h.

Finally, the carpenter comes back to the kitchen, the tension between him and the plumber resumes. The carpet fitter is still quite nervous, as he has to begin his work with 
stricter schedule than he expected. The electrician is still anxious about the very possible necessity of rework. This final state is shown in Fig. 4 i.

The consequences are predictable - "the carpet fitter charges for time wasted, and also, even though the electrician is competent and experienced, he misses out a part of his work in the bathroom" [3], "the final delivery of the contract got squeezed, and so more staff were called in, resulting in overcrowding - so slower working, and having to learn about both house and contract - time wasted, and, the guesses made by the carpenter and electrician were wrong and so rework resulted" [3].

\section{Discussion}

"The spring chart" provides insight into the inclination from the initial schedule when deciding on changes. For instance, if in the project shown in Fig. 3 the further reductions in the activity duration are necessary, it will be easy to see from the last graph (Fig. 3 bottomright) that Activities 3 and 4 are reduced most strongly, and it might be more rational to compress Activity 2 or even 5.

Besides, comparing the graphs obtained as a result of a number of consecutive changes, one can get insight into the dynamics of activities durations' changes. In particular, it can facilitate identification of the most volatile activities (the variability of the expected activity's finish may be caused by frequent occurrence of defects). This is especially important if the similar projects are planned in the future, since studying a "history of spring fluctuation" can contribute to more accurate assessment of the activity duration and risks at the project design stage.

"The spring mapping" allows project managers to understand the current psychological state of stakeholders as well as how it has been changing before. From the example shown in Fig. 4 one can instantly perceive that the situation is far from stable, because all the contractors are stressed (Fig. 4i), and that a disaster is up to appear. One can also see what has led to this state - by tracing the previous graphs. In particular, it is clear that the problem is connected with the carpenter's uneasiness and with the manager's refusal to deal with it. So, a project manager finds out quickly on whose activity he/she should concentrate on.

Worth noting that the examples given are very simple. It is unlikely that making decisions on changes in such cases might cause any problem, even if there is no special visualization tools. The proposed techniques focus mainly on the far more complex projects, such as nuclear power plants construction, in which there are thousands of activities and hundreds of stakeholders. In particular, it is doubtful that "the spring mapping" will be applicable at the operational level, but at the tactic and strategic levels, in which not individuals but organizations are considered as stakeholders, it seems promising. The simple examples are given here only for illustrative purposes.

Although the proposed techniques are apparently more suitable for managing changes, they can't replace the existing techniques in every case. For instance, "the spring chart" is much "heavier" representation than the Gantt chart with simple bars. So, to ease perception when the activity's inclination from the initial schedule doesn't matter, it seems appropriate to create the special software tools allowing a user to switch from one type to another. "The spring mapping" may be too laconic in comparison with the existing techniques like "The Stakeholder Circle". In order to add another data about stakeholders, the special interface with drop-down boxes and hyperlinks might be useful. Besides, the integral techniques should be developed, e.g., drawing "the springs-stakeholders" right upon the sectors in "The Stakeholder Circle".

In addition to the ways of further research mentioned above (related to the software development and the integration with the other techniques), it is necessary to test the new 
techniques in real practice. In particular, the correlation should be studied between the geometrical changes in representation and the corresponding changes in perception.

\section{Conclusions}

The main result of the study is two techniques for the visualization of project dynamics. The first technique named "the spring chart" is the Gantt chart modification, which facilitates taking into account previous changes during rescheduling. The second technique named "the spring mapping" is the way to represent stakeholders relationships through depiction of their psychological state. In both techniques the image of spring used in mechanics is applied as a metaphor.

In "the spring chart" springs replace the bars representing activities in the traditional Gantt chart. The springs are "compressed" or "stretched" according to the changes in a schedule. Such representation contributes to intuitive understanding of an activity duration's degree of deviation from the initial schedule. This is important for deciding the duration of which activities should be changed and how much.

In "the spring mapping" technique springs represent stakeholders. A spring "compresses" when a stakeholder is uneasy due to some problem and "relaxes" when there is no problems. The extent of perceived compression is proportional to a stakeholder's psychological tension. This representation facilitates the perception of changes in stakeholders' relationships because it abstracts unnecessary details of the situation and shows only the most important (in terms of project stability) feature - the level of a stakeholder's satisfaction. Simple representation is expected to be especially effective in the complex projects with strict schedule, because making decision on stakeholders management is very difficult there but should be done quickly.

The proposed techniques can improve the quality of decision-making in dynamic construction project environment. It is especially important for complex projects where incorrect decisions may lead to critical consequences. Besides, they can become a convenient tool (a kind of illustration) when analyzing a "project history", which is especially important when the similar projects are planned.

Despite of the techniques' advantage for representing changes, they have the drawbacks related to some visual complexity and lack of data. These drawbacks might be overcome by integration with the existing techniques. Besides, the development of special software is needed to make the techniques' implementation convenient.

\section{References}

1. Q. Hao, W. Shen, J. Neelamkavil, R. Thomas, Proc. of the CIB W78 25th Int. Conf. on Inf. Tech.: Improv. the Man.t of Constr. Proj. Thr. IT Adop. (Santiago, Chile, July 1517, 2008), 387-396 (2008)

2. S. Kermanshachi, R. Thakur, P. Govan, Proc. of Constr. Res. Congr. (New Orleans, Louisiana, April 2-4, 2018), 691-701 (2018)

3. C. Eden, T. Williams, F. Ackermann, S. Howick, J. Op. Res. Soc., 51(3), 291-300 (2000)

4. T. Taylor, D. Ford, S. Johnson, Proc. of the 23th Int. Conf. of the Syst. Dyn. Soc. (Boston, MA, July 17-21, 2005) (2005)

5. S.-S. Liu, K.-C. Shin, Aut. in Con., 18(6), 715-723 (2009)

6. J. B. H. Yap, H. Abdul-Rahman, Ch. Wang, J. Surv., Con. \& Prop., 8(1), 13-33 (2017)

7. G. Zhu, J. F. Bard, G. Yu, J. Op. Res. Soc., 56, 365-381 (2005)

8. R. Atkinson, L. H. Crawford, S. Ward, Int. J. Pr. Man., 24, 687-98 (2006)

9. P. Anderson, Org. Sci., 10(3), 216-232 (1999) 
10. A. Lapidus, K. Tolstova, Ind. \& Civ. Eng., 5, 68-71 (2016)

11. B. Pergamenshchik, V. Undozjorov, E3S Web of Conf. $22^{\text {nd }}$ Int. Sci. Conf. on Con. the Form. of Liv. Env., FORM 2019, 06020 (2019)

12. A. A. Morozenko, Ind. \& Civ. Eng., 4, 41-42 (2013)

13. Y. A. Olawale, M. Sun, Constr. Man. \& Econ., 28, 509-526 (2010)

14. R. Lengler, M. J. Eppler, Proc. of Graph.s and Vis. in Eng. (GVE, Clearwater, Florida) (2007)

15. V. D. Kolychev and V. P. Rumyantsev, Sci. Vis., 6(3), 14-54 (2014)

16. M. Hajdu, S. Isaac, Org., Tech. \& Man. Con., 8, 1499-1510 (2016)

17. D. H. T. Walker, L. M. Bourne, A. Shelley, Org., Tech. \& Man. Con., 8, 1499-1510 (2016)

18. L. Bourne, D. H. T. Walker, Man. Dec., 43(5), 649-660 (2005)

19. A. Shelley, The organizational zoo: a survival guide to workplace behavior, Connecticut USA, Aslan Publishing (2007)

20. R. Albuquerque, M. Eichenbaum, D.Papanikolaou, S. Rebelo, J. Mon. Econ., 76:S21 S36, (2015)

21. S. Eijffinger, R. Mahieu, L. Raes, CEPR disc. papers, DP9418 (2013) 